\title{
Dissertationen der Doktoranden von Prof. Dr. Heinrich Buess, Universität Basel
}

Basler Veröff. $=$ Basler Veröffentlichungen zur Geschichte der Medizin und der Biologie, herausgegeben von Prof.Dr.med.H.Buess, Schwabe Verlag, Basel (ab Fasc. VI: Basel/ Stuttgart).

1. Sigmund Bornhauser, Zur Geschichte der Schilddrüsen- und Kropfforschung im 19. Jahrhundert (unter besonderer Berücksichtigung der Schweiz). (Veröffentlichungen der Schweizerischen Gesellschaft für Geschichte der Medizin und der Naturwissenschaften, Bd. XIX, Verlag Sauerländer, Aarau 1951.)

2. Beate Moeschlin-Krieg, Zur Geschichte der Regenerationsforschung im 18. Jahrhundert (Basler Veröff., Fasc. I, 1953).

3. Irma Steiner, Die geschichtliche Entwicklung der chemischen Mutterkornforschung bis zum Jahre 1918, Basel 1951.

4. Benno Flüeler, Ärzte, Apotheker, Chirurgen und Hebammen im alten Stande Solothurn 1481-1798. Ein Beitrag zur Geschichte des Medizinalwesens in der Schweiz. Solothurn 1951 (Jahrbuch für solothurnische Geschichte, Bd. 24).

5. Eva Lichtenberg, Die Geschichte der intravenösen Injektion im 19. Jahrhundert, Wien 1952.

6. Philipp Eyholzer, Geschichtliches zur Therapie der perforierenden Darmverletzung. Von den Anfängen bis zu Beginn des 18. Jahrhunderts. Zürich 1950.

7. Hans Rudolf Thüer, Johann Balthasar Luchsinger (1849-1886). Ein wenig bekannter Schweizer Physiologe (Basler Veröff., Fasc. II, 1953).

8. Ernst Rolf Buser, Zur Entwicklung des Badewesens im Unterengadin. Ein Beitrag zur Geschichte der Balneologie in der Schweiz (Basler Veröff., Fasc. IV, 1954).

9. Erich Auer, Entwicklung und Stand der sozialmedizinischen Reform in der schweizerischen Industrie. Ein Beitrag zur Geschichte der Arbeitshygiene. Diss. med. dent. Bern (erschienen in: Basler Veröff., Fasc. V, 1955).

10. Nikolaus Mani, Die historischen Grundlagen der Leberforschung. I.Teil. Die Vorstellungen über Anatomie, Physiologie und Pathologie der Leber in der Antike (Basler Veröff., Fasc. IX, 1959). Mit Fortsetzung: II. Teil. Die Geschichte der Leberforschung von Galen bis Claude Bernard (Basler Veröff., Fasc. XXI, 1967).

11. Max Winzenried, Das Militärsanitätswesen in der Schweiz von der Mediation bis zum Sonderbundskrieg. Diss. med. dent. Bern (erschienen in: Basler Veröff., Fasc. III, 1954).

12. Marianne Schorr, Zur Geschichte der Bluttransfusion im 19. Jahrhundert. Unter besonderer Berücksichtigung ihrer biologischen Grundlagen (Basler Veröff., Fasc. VII, 1956).

13. Armando Andreoli, Zur geschichtlichen Entwicklung der Neuronentheorie (Basler Veröff., Fasc. X, 1961).

14. Susi Joos-Renfer, Pathologisch-anatomische Beobachtungen bedeutender Schweizer Ärzte 1670-1720. Diss. med. dent. Bern (erschienen in: Basler Veröff., Fasc. XIII, 1961).

15. Peter Molling, Theodor Zwinger d. J. (1658-1724) als Kinderarzt (Studien zur Geschichte der Wissenschaften in Basel, Bd. XIV, Basel 1962). 
16. Johannes Gartmann, Johann Georg Amstein (1744-1794). Ein gelehrter Bündner Arzt und Naturforscher des 18. Jahrhunderts (Basler Veröff., Fasc. VI, 1956).

17. Hans Kräuchi, Über die Wandlungen der wichtigsten Grundsätze in der Kriegschirurgie und Sanitätstaktik des 19. Jahrhunderts, Basel 1955.

18. Mario Studer, Das amtliche Medizinalwesen im alten Luzern, unter besonderer Berücksichtigung der Stadtärzte und ihrer Pflichten, Stans 1958 (aus: Der Geschichtsfreund, Bd. 111).

19. Friedrich Huber, Daniel Bernoulli (1700-1782) als Physiologe und Statistiker (Basler Veröff., Fasc. VIII, 1958).

20. Paul Popp, Der Beitrag von Jakob Laurenz Sonderegger (1825-1896) zur Sozialmedizin und Sozialpolitik, Zürich 1960.

21. Jean-Pierre Colombo, Heinrich Bircher (1850-1923), Chirurg, Demograph und Militärarzt (Basler Veröff., Fasc. XII, 1961).

22. Edmond Shepper, Ludwig Georg Courvoisier (1843-1918) and his Position in the History of Gall Bladder Surgery, Basel 1960.

23. Charles Abraham Greene, The Contributions of Rudolph Virchow (1821-1902) to Obstetrics and Gynaecology, Basel 1960.

24. Werner Stöcklin, Der Basler Arzt Leopold Rütimeyer (1856-1932) und sein Beitrag zur Ethnologie (Basler Veröff., Fasc. XI, 1961).

25. Otto Mandler, Johann Jacob Bischoff (1841-1892) und die Anfänge moderner Geburtshilfe in Basel, Bern 1960 (aus: Praxis. Schweizerische Rundschau für Medizin, 49. Jg., No. 41 und 42).

26. Julius Reiner, Der Beitrag von Friedrich Miescher d.J. (1844-1895) zur Geschichte der Zellbiologie, Basel 1963.

27. Manfred Frey, Friedrich Miescher-His (1811-1887) und sein Beitrag zur Histopathologie des Knochens (Basler Veröff., Fasc. XIV, 1962).

28. Karl Flachsmann, Der Engadiner Arzt Oskar Bernhard (1861-1939) und die Begründung der Heliotherapie bei der chirurgischen Tuberkulose (Basler Veröff., Fasc. XXII, 1966).

29. Michael Hammer, Über den Beitrag der ersten Wiener Ärzte-Schule zur Arzneimittellehre und prophylaktischen Medizin, Winterthur 1962.

30. Edgar Widmer, Zur Geschichte der schweizerischen ärztlichen Mission in Afrika unter besonderer Berücksichtigung des medizinischen Zentrums von Ifakara, Tanganyika (Basler Veröff., Fasc. XVI, 1963).

31. Christian Hodel, Die Anfänge der Krankenversicherung in Basel während des 19. Jahrhunderts und ihre geschichtlichen Voraussetzungen (Basler Veröff., Fasc. XX, 1965).

32. Pietro Eichenberger, Johann Jakob Wepfer (1620-1695) als klinischer Praktiker (Basler Veröff., Fasc. XXVI, 1969).

33. Reinhold Christian Link, Über die Multiplizität primärer maligner Geschwülste beim beruflichen malignen Harnblasentumor, Basel 1961.

34. Hans-Rudolf Haller, Gustav Wolf (1865-1941) und sein Beitrag zur Lehre vom Vitalismus (Basler Veröff., Fasc. XXIV, 1968).

25. Eduard Bider, Alfred Vogt in Basel, 1918-1923 (Basler Veröff., Fasc. XVIII, 1964).

36. Dietegen Guggenbühl, Gerichtliche Medizin in Basel von den Anfängen bis zur Helvetik (Basler Veröff., Fasc. XV, 1963). 
37. Howard Charles Adelmann, Percival Pott's Role in the History of Surgery and Fracture Therapy, Basel 1963.

38. Ernst Meyer, Friedrich Trendelenburg (1844-1924) und seine Verdienste um die Beckenhochlagerung, Basel 1964.

39. Matthew M.Quinones, Robert Adams and his Role in the History of Rheumatoid Arthritis, Basel 1966.

40. Peter Wobmann, Albrecht von Haller, der Begründer der modernen Hämodynamik, Darmstadt 1967 (aus: Archiv für Kreislaufforschung, Bd. 52).

41. Hans Häfliger, Zur Geschichte der Hämophilie unter besonderer Berücksichtigung der Schweiz (Basler Veröff., Fasc. XXVIII, 1969).

42. Paul Morris Dlugatz, Astley Cooper's Contribution to the Knowledge of Dislocations and Fractures, Zürich 1968.

43. Paul Johann Dubler, Johann Jakob Baader (1810-1879), Arzt und Politiker in Gelterkinden, Liestal 1970 (Quellen und Forschungen zur Geschichte und Landeskunde des Kantons Baselland, Bd. X).

44. Ali Reza Moshfegh, Carl Sebastian Haegler (1862-1916) und sein Beitrag zur Geschichte der Aseptik, Basel 1970.

45. Osman Karamehmedovic, Ernst Tavel (1858-1912), Bakteriologe und Chirurg in Bern (Berner Beiträge zur Geschichte der Medizin und der Naturwissenschaften, Neue Folge, Bd. 7, Verlag Hans Huber, Bern/Stuttgart/Wien 1973).

4.6. Eleonore Knüsel, Über die Anfänge der pathologischen Anatomie und ihrer Historiographie, Basel 1969.

47. Dietmar Tetzlaff, Johannes von Mikulicz-Radecki (1850-1905) und die Herausbildung der aseptischen Hilfsmittel, Basel 1971.

48. Monika Fuchs-Wy $\beta$, Jakob Laurenz Sonderegger (1825-1896) als Standespolitiker, Basel 1971.

49. Alois Lang, Albert Burckhardt-Merian (1843-1886) als Mitbegründer der modernen Ohrenheilkunde, Basel 1971.

50. Rudolf Andreas Elliker, Die Behandlung der Frakturen in der Chirurgie der Barockzeit, aufgezeigt am Beispiel von Wilhelm Fabry von Hilden (1560-1634), Basel 1972.

51. Jeannette Hofer-Eisenring, Zur Geschichte der Regenerationsforschung im 19.Jahrhundert unter besonderer Berücksichtigung des Muskelgewebes, Basel 1972.

52. Peter Brin, J.F.Malgaigne (1806-1865). Sein Beitrag zur Lehre von den Frakturen. Basel 1972.

53. Emilia Voegelin-Raess, Zur Einführung der Inhalationsnarkose in der Schweiz, Basel 1973.

54. Gerhard Schmidt, Das geistige Vermächtnis von Gustav v. Bunge, Zürich 1973.

55. Monika Baltzer, Die Vorläufer der Medizinischen Gesellschaft Basel (in der ersten Hälfte des 19. Jahrhunderts), Basel 1974.

56. Rolf Aschwanden, Zur Sexual- und Ehe-Hygiene des Hippolytus Guarinonius (1571 bis 1654) anhand seines Werkes «Die Grewel der Verwüstung Menschlichen Geschlechts», Basel 1974.

57. Regina Egger, Archivalische Grundlagen zur Entwicklung der Medizinischen Poliklinik in Basel, Basel 1975. 
58. Dominik Leupold, Das Handauflegen, eine ärztliche Urgebärde in Geschichte und Gegenwart, Basel 1975.

59. Fritz Ramseier, Die neolithische, bronzezeitliche und eisenzeitliche Schädeltrepanation in der Schweiz, Basel 1976.

60. Paul Gasser, Charles Krafft (1863-1921), ein Pionier der Appendektomie und der Krankenpflege in Europa (Basler Veröff., Fasc. XXX, 1977).

61. Gottfried Hofer, Studien zur Geschichte der Regenerationsforschung im neunzehnten Jahrhundert unter besonderer Berücksichtigung der Regenerationsforschung bei Wirbellosen und bei niederen Tieren, Basel 1976.

62. Christof Graf, Radiologische Untersuchungen an sechs altägyptischen Mumien aus dem Museum für Völkerkunde in Basel, Basel 1976.

63. Markus Hämmerle, Die Anfänge der Basler Chemischen Industrie im Lichte von Arbeitsmedizin und Umweltschutz (Basler Veröff., Fasc. XXXII, 1978).

64. Melitta Bosshart, Zur Pädiatrie im Corpus Hippocraticum, Basel 1977.

65. Hanspeter Rölli, Vom Beitrag der Basler Klinik zur Überwindung des Therapeutischen Nihilismus, Basel 1977.

66. Anton Treier, Theodor Zwinger (1658-1724) und sein Hausarzneibuch, Basel 1977.

67. Jürg Karlen, Ausgewählte Daten aus der neueren Geschichte des Experimentes am Menschen. Ein Beitrag zur Geschichte der klinischen Pharmakologie. Basel 1977.

Jakob Tschudi und Marie-Louise Portmann 